\title{
A reservoir of timescales in random neural networks
}

\author{
Merav Stern ${ }^{a, b, 1}$, Nicolae Istrate ${ }^{a, c, 1}$, and Luca Mazzucato ${ }^{a, c, d}$ \\ ${ }^{a}$ Institute of Neuroscience, ${ }^{c}$ Departments of Physics, \\ ${ }^{d}$ Mathematics and Biology, \\ University of Oregon, Eugene. \\ ${ }^{b}$ Faculty of Medicine, \\ The Hebrew University of Jerusalem, Jerusalem. \\ ${ }^{1}$ equal contribution
}

\begin{abstract}
The temporal activity of many biological systems, including neural circuits, exhibits fluctuations simultaneously varying over a large range of timescales. The mechanisms leading to this temporal heterogeneity are yet unknown. Here we show that random neural networks endowed with a distribution of self-couplings, representing functional neural clusters of different sizes, generate multiple timescales activity spanning several orders of magnitude. When driven by a time-dependent broadband input, slow and fast neural clusters preferentially entrain slow and fast spectral components of the input, respectively, suggesting a potential mechanism for spectral demixing in cortical circuits. occur.
\end{abstract}

\section{INTRODUCTION}

Experimental evidence shows that the temporal activity of many physical and biological systems exhibits fluctuations simultaneously varying over a large range of timescales. In condensed matter physics for example, spin glasses typically exhibit aging and relaxation effects whose timescales span several orders of magnitude [1]. In biological systems, metabolic networks of E. coli generate fluxes rate with a power-low distribution $[2,3]$. And yeast cultures release gases in frequency distributions that reveal activity across many orders of magnitude [4], endowing them with robust and flexible responses to the environment [5].

In the mammalian brain, a hierarchy of timescales in the activity of single neurons is observed across different cortical areas from occipital to frontal regions [6-8]. Moreover, neurons within the same local circuit exhibit a large range of timescales from milliseconds to minutes $[9,10]$. This heterogeneity of neuronal timescales was observed in awake animals during periods of ongoing activity, in the absence of external stimuli or behavioral tasks, suggesting that multiple timescales of neural activity may be an intrinsic property of recurrent cortical circuits. Recent studies highlighted the benefits of leveraging computations on multiple timescales when performing complex tasks in primates [11] as well as in artificial neural networks [12]. However, the neural mechanisms underlying the emergence of multiple timescales are not yet understood. We suggest here such mechanism.

We focus on random neuronal networks whose units are recurrently connected, with couplings that are chosen randomly. In our model, each network unit represents a functional cluster of cortical neurons with similar response properties. We interpret the unit's self-coupling as evident to the cluster size and the recurrent coupling strength of its neurons. In the case where the selfcouplings are zero or weak (order $1 / \sqrt{N}$ ), random networks are known to undergo a phase transition from si- lence to chaos when the variance of the random couplings exceeds a critical value [13]. When the self-couplings are strong (order 1) and are all equal, a third phase appears featuring multiple stable fixed points accompanied by long transient activity [14]. In all these cases, all network units exhibit the same intrinsic timescale, estimated from their autocorrelation function.

Here, we demonstrate a novel class of recurrent networks, capable of generating temporally heterogeneous activity whose multiple timescales span several orders of magnitude. We show that when the self-couplings are heterogeneous, a reservoir of multiple timescales emerges, where each unit's intrinsic timescale depends both on its own self-coupling and the network's self-coupling distribution. We analytically study the dynamics of a single unit in the limit of large self-coupling, revealing a new metastable regime described by colored noise-driven transitions between potential wells. We also study the stimulus-response properties of our networks with heterogeneous self-couplings. In networks with zero or weak self-couplings chaotic activity is suppressed best at a single resonant frequency [15]. However, when we drive our networks with a time-dependent broadband input featuring a superposition of multiple frequencies, we find that the chaotic activity is suppressed across multiple frequencies which depend on the units' respective selfcouplings. We see that units with large and small selfcouplings are preferentially entrained by the low and high frequency components of the input, respectively. This spectral specificity suggests that a reservoir of timescales may be a natural mechanism for cortical circuits to flexibly demix different spectral features of complex timevarying inputs.

Random networks with heterogeneous selfcouplings. We consider a recurrent network of $N$ rate units obeying the dynamical equations

$$
\frac{d x_{i}}{d t}=-x_{i}+s_{i} \phi\left(x_{i}\right)+g \sum_{j=1}^{N} J_{i j} \phi\left(x_{j}\right)
$$




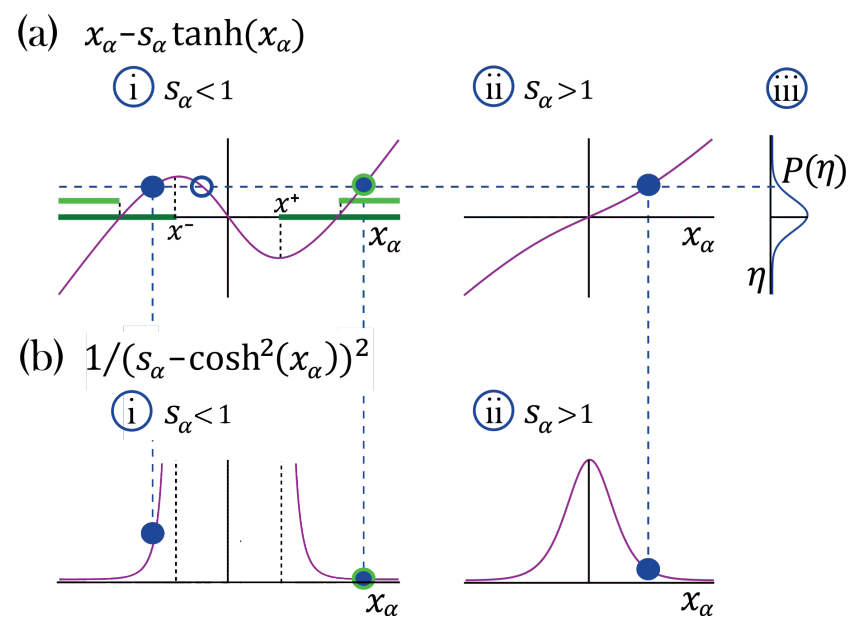

FIG. 1. Transition to chaos with multiple self-couplings: Fixed point solutions and stability. a-i) The fixed point curve $x_{\alpha}-s_{\alpha} \tanh x_{\alpha}$, from Eq. (4), for $s_{\alpha}>1$. Stable solutions are allowed within the dark green region. b-i) The shape of a unit's contribution to stability $q^{-1}=\left(s_{\alpha}-\cosh x_{\alpha}^{2}\right)^{-2}$, from Eq. (7). Stable solutions of $x_{\alpha}-s_{\alpha} \tanh x_{\alpha}=\eta$, filled blue circles in (a-i), with different $|x|$ values contribute differently to stability. At the edge of chaos only a fixed point configuration with all units contributing most to stability (minimal $q^{-1}$ ) is stable, light green region in (a-i). a-ii) The curve $x_{\alpha}-s_{\alpha} \tanh x_{\alpha}$ for $s_{\alpha}<1$. a-iii) A possible distribution of the Gaussian mean-field $\eta$. A representative fixed point solution is illustrated by the dashed blue line: for $s_{\alpha}<1$ a single solution exists for all values of $\eta$, (filled blue circle in a-ii);For $s_{\alpha}>1$ multiple solutions exist (a-i) for some values of $\eta$; some of them lead to instability (empty blue circle in a-i). The other two solutions may lead to stability (filled blue circles in a-ii), although only one of them will remain stable at the edge of chaos (encircled with green line in a-i).

where the random couplings $J_{i j}$ from unit $j$ to unit $i$ are drawn independently from a Gaussian distribution with mean 0 and variance $1 / N ; g$ represents the network gain and we chose a transfer function $\phi(x) \equiv \tanh (x)$. We measure time in units of $1 \mathrm{~ms}$. The self-couplings $s_{i}$ are drawn from a distribution $P(s)$. The special case of equal self-couplings $\left(s_{i}=s\right)$ was studied in [14] and a summary of the results are brought in the Appendix for convenience. Here we study the network properties in relation to both discrete and continuous distributions $P(s)$.

Using standard methods of statistical field theory $[16,17]$, in the limit of large $N$ we can average over realizations of the disordered couplings $J_{i j}$ to derive a set of self-consistent dynamic mean field equations for each population of units $x_{\alpha}$ with self-coupling strength $s_{\alpha} \in S$

$$
\frac{d x_{\alpha}}{d t}=-x_{\alpha}+s_{\alpha} \tanh \left(x_{\alpha}\right)+\eta(t) .
$$

In our notation, $S$ denotes the set of different values of self-couplings $s_{\alpha}$, indexed by $\alpha \in A$, and we denote by $N_{\alpha}$ the number of units with the same self-coupling $s_{\alpha}$, and accordingly by $n_{\alpha}=N_{\alpha} / N$ their fraction. The mean field $\eta(t)$ is the same for all units and has zero mean $\langle\eta(t)\rangle=0$ and autocorrelation

$$
\begin{aligned}
& \langle\eta(t) \eta(t+\tau)\rangle=g^{2} C(\tau) \\
& C(\tau)=\sum_{\alpha \in A} n_{\alpha}\left\langle\phi\left[x_{\alpha}(t)\right] \phi\left[x_{\alpha}(t+\tau)\right]\right\rangle,
\end{aligned}
$$

where $\langle\cdot\rangle$ denotes an average over the mean field.

Stable fixed-points and transition to chaos. Networks with heterogeneous self-couplings exhibit a complex landscape of fixed points $x_{\alpha}^{*}$, obtained as the selfconsistent solutions to the static version of Eq. (2) and Eq. (3), namely

$$
x_{\alpha}-s_{\alpha} \tanh \left(x_{\alpha}\right)=\eta,
$$

where the mean field $\eta$ has zero mean and variance

$$
\begin{aligned}
\left\langle\eta^{2}\right\rangle & =g^{2} C \\
C & =\sum_{\alpha \in A} n_{\alpha}\left\langle\phi\left[x_{\alpha}\right]^{2}\right\rangle .
\end{aligned}
$$

The solution for each unit depends on its respective $s_{\alpha}$ (Fig. 1). If $s_{\alpha}<1$ a single interval around zero is available. For $s_{\alpha}>1$, for a range of values of $\eta, x_{\alpha}^{*}$ can take values in one of three possible intervals. However, the available solutions in the latter case are further restricted by stability conditions.

We can derive the stability condition by expanding the dynamical equations (1) around the fixed point and requiring that all eigenvalues of the corresponding stability matrix are negative. An eigenvalue of the stability matrix exists at a point $z$ in the complex plain if $[14,18]$

$$
g^{2} \sum_{\alpha \in A} n_{\alpha}\left\langle\frac{\left[1-\tanh ^{2}\left(x_{\alpha}\right)\right]^{2}}{\left[z+1-s_{\alpha}\left(1-\tanh ^{2}\left(x_{\alpha}\right)\right)\right]^{2}}\right\rangle>1 .
$$

Since the denominator of the expression above is $z$ plus the slope of the curve in Fig. 1a-i, a solution whose value $x_{\alpha}^{*}$ gives a negative slope (available when $s_{\alpha}>1$ ) leads to a vanishing value of the denominator at some positive $z$ and hence to a positive eigenvalue and instability. Hence, the $n_{\alpha}$ fraction of units with $s_{\alpha}>1$ at a stable fixed point are restricted to have support on two disjoint intervals $\left[x_{\alpha}^{*}\left(s_{\alpha}\right)<x_{\alpha}^{-}\left(s_{\alpha}\right)\right] \cup\left[x_{\alpha}^{*}\left(s_{\alpha}\right)>x_{\alpha}^{+}\left(s_{\alpha}\right)\right]$. We refer to this regime as multi-modal, a direct generalization of the stable fixed points regime found in [14] for a single self-coupling $s>1$, characterized by transient dynamics leading to an exponentially large number of stable fixed points. For the $n_{\alpha}$ portion of units with $s_{\alpha}<1$, the stable fixed point is supported by a single interval around zero.

A fixed point solution becomes unstable as soon as an eigenvalue occurs at $z=0$, obtaining from Eq. (6) the stability condition

$$
g^{2} \sum_{\alpha \in A} n_{\alpha}\left\langle q_{\alpha}^{-1}\right\rangle \leq 1
$$

where $q_{\alpha}=\left[s_{\alpha}-\cosh ^{2}\left(x_{\alpha}\right)\right]^{2}$. For $s_{\alpha}>1$ the two possible consistent solutions to (4) that may result in a stable 
(a) $s_{1}$

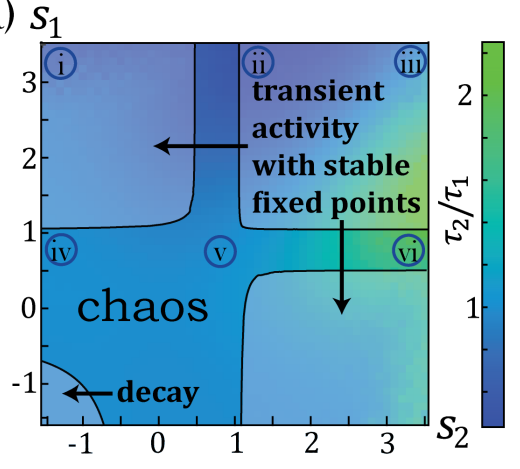

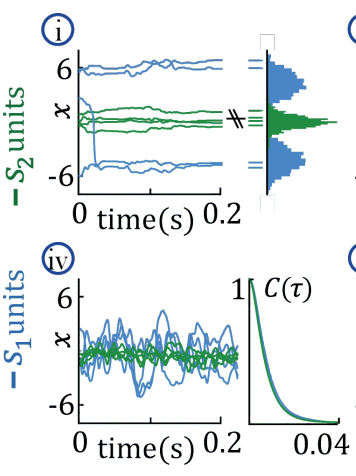

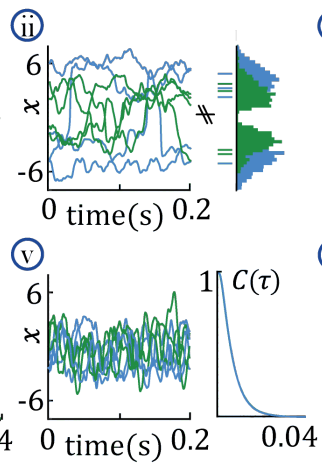

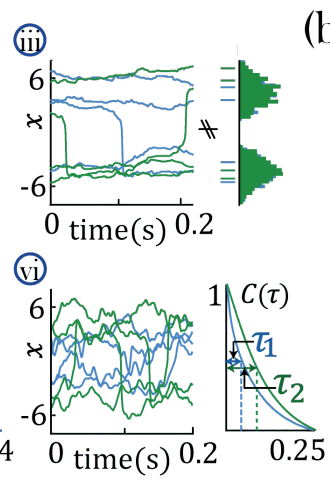

FIG. 2. Dynamical and fixed point properties of networks with two self-couplings. a) Ratio of autocorrelation timescales $\tau_{2} / \tau_{1}$ of units with self-couplings $s_{2}$ and $s_{1}$, respectively $\left(\tau_{i}\right.$ is estimated as the half width at half max of a unit's autocorrelation function, see panels iv-vi), in a network with $n_{1}=n_{2}=0.5$ and $g=2$ and varying $s_{1}, s_{2}$. A central chaotic phase separates four different stable fixed point regions with or without transient activity. Black curves represent the transition from chaotic to stable fixed point regimes. i)-iii) Activity across time during the initial transient epoch (left) and distributions of unit values at their stable fixed points (right), for networks with $N=1000$ and (i) $s_{1}=3.2, s_{2}=-1.5$, (ii) $s_{1}=3.2, s_{2}=1.2$, (iii) $s_{1}=3.2, s_{2}=3.2$. iv) - vi) Activity across time (left) and normalized autocorrelation functions $C(\tau) / C(0)$, (right) of units with (iv) $s_{1}=0.8, s_{2}=-1.5$, (v) $s_{1}=0.8, s_{2}=0.8$, (vi) $s_{1}=0.8, s_{2}=3.2$. b) Timescale ratio $\tau_{2} / \tau_{1}$ for fixed $s_{1}=1$ and varying $s_{2}$.

fixed point (from the two disjoint intervals in Fig. 1a-i), contribute differently to $q_{\alpha}$. Larger $\left|x_{\alpha}^{*}\right|$ decreases $q_{\alpha}^{-1}$ (Fig. 1b-i), thus improving stability. Choices for distributions of $x_{\alpha}^{*}$ along the two intervals become more restricted as $g$ increases or $s_{\alpha}$ decreases, since both render higher values for the stability condition, Eq. 7, forcing more solutions of $x_{i}$ to decrease $q_{\alpha}^{-1}$. This restricts a larger fraction of $x_{\alpha}^{*}$ at the fixed points to the one solution with higher absolute value. At the transition to chaos, a single last and most stable solution exists with all $x_{i}$ values chosen with their higher absolute value $x_{\alpha}^{*}$ (Fig. 1a-i, light green segments). For those with $s_{\alpha}<1$ only one solution is available, obtained by the distribution of $\eta$ through consistency (4) at the fixed point. In this configuration, the most stable solution is exactly transitioning from stability to instability where (7) reaches unity. Hence the transition from stable fixed points to chaos occurs for a choice of $g$ and $P(s)$ such that solving consistently (4) and (5) leads to saturate the stability condition (7) at one.

We illustrate the discussion above in the case of a network with two sub-populations with $n_{1}$ and $n_{2}=1-n_{1}$ portions of the units with self-couplings $s_{1}$ and $s_{2}$, respectively. In the $\left(s_{1}, s_{2}\right)$ plane, this model gives rise to a phase portrait with a single chaotic region separating four disconnected stable fixed-point regions (Fig. 2a). A unit's activity is determined by its own self-coupling, the network's distribution of self-couplings and $g$. When both self-couplings are greater than one $\left(s_{1}, s_{2}>1\right)$ the fixed point distribution in each sub-population is bi-modal (Fig. 2a-ii,iii). When $s_{1}>1$ and $s_{2}<1$, the solutions for the respective sub-populations are localized around bi-modal fixed points and around zero, respectively (Fig. 2a-i). When both $s_{1}, s_{2}<1$, the only possibility for a stable fixed point is the trivial solution, with all $x_{i}=0$ (Fig. 2a, decay region). In the case of a Gaussian distribution of self-couplings in the stable fixed point regime, a complex landscape of stable fixed points emerges which continuously interpolates between the zero (for units with $s_{i}<1$ ) and the multiple values bi-modal cases (for units with $s_{i}>1$ ) within the same network (Fig. 3a).

A reservoir of timescales. In the chaotic phase we can estimate the intrinsic timescale $\tau_{i}$ of a unit $x_{i}$ from its autocorrelation function $C(\tau)=\left\langle\phi\left[x_{i}(t)\right] \phi\left[x_{i}(t+\tau)\right]\right\rangle_{t}$ as the half width at its autocorrelation half maximum (Fig. 2a-vi, $\tau_{1}$ and $\left.\tau_{2}\right)$. The chaotic phase in the network, Eq. (1), is characterized by a large range of timescales that can be simultaneously realized across the units with different self-couplings. As a first example, in the network with two self-couplings, we found a parametric separation of timescales between the two sub-populations $s_{1}$ and $s_{2}$ in the chaotic region, where the ratio of timescales $\tau_{2} / \tau_{1}$ is a supra-linear function of the self-couplings ratio $s_{2} / s_{1}$ (Fig. $2 \mathrm{~b}$ ).

In a case of a lognormal distribution of self-couplings, in the chaotic regime the network generates a reservoir of multiple timescale $\tau_{i}$ of chaotic activity across network units, spanning across several orders of magnitude (Fig. $3 \mathrm{~b}$ ). For long tailed distributions such as the lognormal, mean field theory can generate predictions for rare units with large self-couplings from the tail end of the distribution by solving (2) and the continuous version of (3), see Appendix, highlighting the exponential relation between a units self-coupling and its autocorrelation decay time.

Separation of timescales in the bistable chaotic 
(a) At a Fixed Point

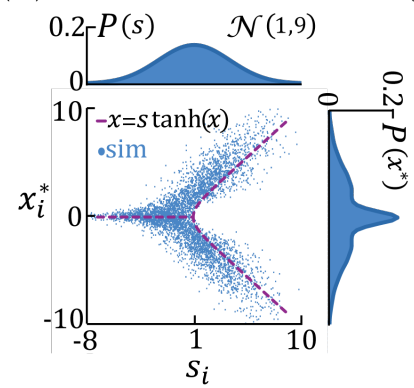

(b) During Chaotic Activity

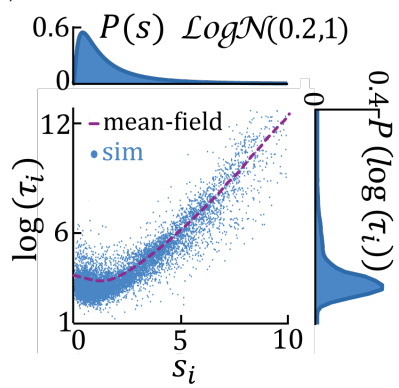

FIG. 3. Continuous distributions of self-couplings. a) In a network with a Gaussian distribution of self-couplings (mean $\mu=1$ and variance $\sigma^{2}=9$ ), the stable fixed point regime exhibits a distribution of fixed point values interpolating between around the zero fixed point (for units with $s_{i} \leq 1$ ) and the multi-modal case (for units with $s_{i}>1$ ). The purple curve represents solutions to $x=s \tanh (x)$. b) In a network with a lognormal distribution of self-couplings (parameters $\mu=0.2$ and $\sigma^{2}=1$ ), autocorrelation timescales $\tau_{i}$ in the chaotic phase span several orders of magnitude as functions of the units' self-couplings $s_{i}$ (purple curve shows the dynamic mean-field predictions for $\tau_{i}$ ).

regime. To gain an analytical understanding of the parametric separation of timescales in networks with heterogeneous self-couplings, we consider the special case of a network with two self-couplings where a large subpopulation $\left(N_{1}=N-1\right)$ with $s_{1}=1$ comprises all but one slow probe unit, $x_{2}$, with large self-coupling $s_{2} \gg s_{1}$. The probe unit obeys the dynamical equation $d x_{2} / d t=f\left(x_{2}\right)+\eta(t)$, with $f(x)=-x+s_{2} \phi(x)$. In the large $N$ limit, we can neglect the backreaction of the probe unit on the mean field and approximate the latter as an external Gaussian colored noise $\eta(t)$ with autocorrelation $g^{2} C(\tau)=g^{2}\left\langle\phi\left[x_{1}(t)\right] \phi\left[x_{1}(t+\tau)\right]\right\rangle$, independent of $x_{2}$. The noise $\eta(t)$ can be parameterized by its strength, defined as $D=\int_{0}^{\infty} d \tau C(\tau)$ and its timescale (color) $\tau_{1}$. For large $s_{2}$, the dynamics of the probe unit $x_{2}$ can be captured by a bi-stable chaotic phase whereby its activity is localized around the critical points $x_{2}=x^{ \pm} \simeq \pm s_{2}$ (Fig. 4a-i) and switches between them at random times. In the regime of strong colored noise (as we have here, with $\tau_{1} \simeq 7.9 \gg 1$ ), the stationary probability distribution $p(x)$ (for $x \equiv x_{2}$, Fig. 4a-ii,b) satisfies the unified colored noise approximation to the Fokker Planck equation $[19,20]$ :

$$
p(x)=Z^{-1}|h(x)| \exp \left[-U_{e f f}(x) / D\right],
$$

where $Z$ is a normalization constant, $h(x) \equiv 1-\tau_{1} f^{\prime}(x)$, and the effective potential $U_{\text {eff }}(x)=-\int^{x} f(y) h(y) d y$ is given by

$$
U_{\text {eff }}=x^{2} / 2-s_{2} \log \cosh (x)+\frac{\tau_{1}}{2} f(x)^{2}-U_{\text {min }} .
$$

The distribution $p(x)$ has support in the region $h(x)>$ 0 comprising two disjoint intervals $|x|>x_{c}$ where $\tanh \left(x_{c}\right)^{2}=1-\frac{1+\tau_{1}}{\tau_{1} s_{2}}$ (Fig. 4b). $p(x)$ is concentrated around the two minima $x^{ \pm} \simeq \pm s_{2}$ of $U_{\text {eff }}$. The main effect of the strong color $\tau_{1} \gg 1$ is to sharply decrease the variance of the distribution around the minima $x^{ \pm}$. This is evident from comparing the colored noise with a white noise, when the latter is driving the same bi-stable probe $d x_{2} / d t=-x_{2}+s_{2} \phi\left(x_{2}\right)+\xi(t)$, where $\xi(t)$ is a white noise with an equivalent strength to the colored noise, Fig. 4a-iv,v,vi. The naive potential for the white noise case $U=x^{2} / 2-s_{2} \log \cosh (x)$ is obtained from (8) by sending $\tau_{1} \rightarrow 0$ in the prefactor $h$ and in potential $U_{\text {eff }}$. It results in wider activity distribution compared to our network generated colored noise, in agreement with the simulations, Fig. 4a,b.

In our network generated colored noise the probe unit's temporal dynamics is captured by the mean first passage time $\langle T\rangle$ for the escape out of the potential well:

$$
\begin{aligned}
\langle T\rangle & =\int_{-s_{2}}^{-x_{c}} \frac{d x}{D} \frac{h(x)^{2}}{p(x)} \int_{-\infty}^{x} p(y) d y \\
& \simeq 2 \pi \sqrt{U_{e f f}^{\prime \prime}\left(x_{-}\right) \rho^{\prime \prime}\left(x_{f}\right)} \exp \left(\frac{\Delta}{D}\right),
\end{aligned}
$$

where $\Delta=\rho\left(x_{f}\right)-U_{\text {eff }}\left(x_{-}\right)$and $\rho=U_{\text {eff }}+D \log h$. We evaluated the integrals by steepest descent around $x^{-}$and $-x_{f}$, where $\tanh \left(x_{f}\right)^{2} \simeq 1-1 / 2 s_{2}$. The agreement between (10) and simulation results improves with increasing $s_{2}$, as expected on theoretical ground $[19,20]$ (Fig. 4c). The asymptotic scaling for large $s_{2}$ is

$$
\log (\langle T\rangle) \sim \frac{\tau_{1}+1}{2 D}\left[s_{2}^{2}-s_{2} \log \left(s_{2}\right)\right] .
$$

In this slow probe regime, we thus achieved a parametric separation of timescales between the population $x_{1}$, with its intrinsic timescale $\tau_{1}$, and the probe unit $x_{2}$ whose activity fluctuations exhibit two separate timescales: the slow timescale $T$ of the bistable switching and the fast timescale $\tau_{1}$ of the fluctuations around the metastable states (obtained by expanding the dynamical equation around the meta-stable values $x^{ \pm}= \pm s_{2}$ ). One can generalize this metastable regime to a network with $N-p$ units which belong to a group with $s_{1}=1$ and $p \ll N$ slow probe units $x_{\alpha}$, for $\alpha=2, \ldots, p+1$, with large self-couplings $s_{\alpha}$. The slow dynamics of each probe unit $x_{\alpha}$ is captured by its own bistable switching time $T_{\alpha}$ in (10) and all slow units are driven by a shared external colored noise $\eta(t)$ with timescale $\tau_{1}$. In summary, in our model multiple timescales can be robustly generated with specific values, varying over several orders of magnitude.

Demixing of time-dependent broadband input. Previous work in random networks with no self-couplings $\left(s_{i}=0\right.$ in (1)) showed that stimulus-driven suppression of chaos is enhanced at a particular input frequency, related to the network's intrinsic timescale [15]. We investigated whether in our network with two different self-couplings $s_{1}<s_{2}$, in the chaotic regime, the stimulus-dependent suppression of chaos exhibited different features in the 

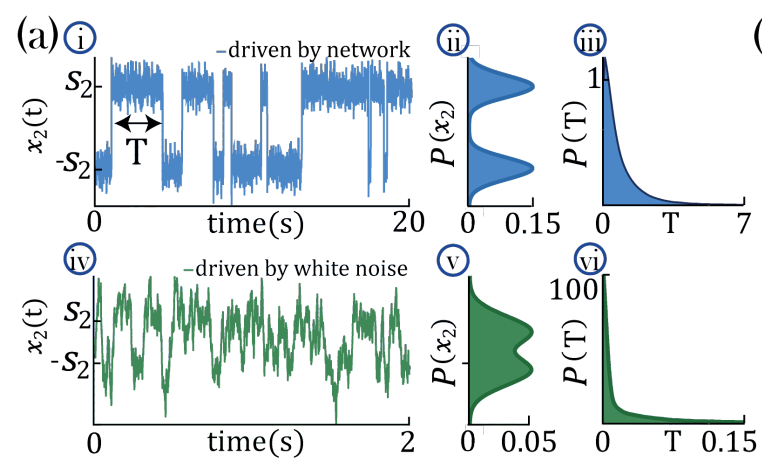

(b)

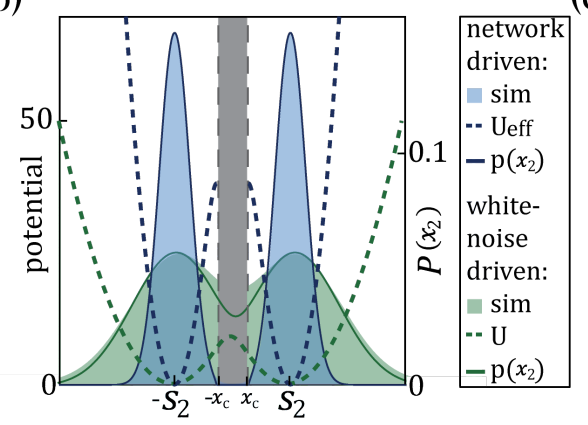

(c)

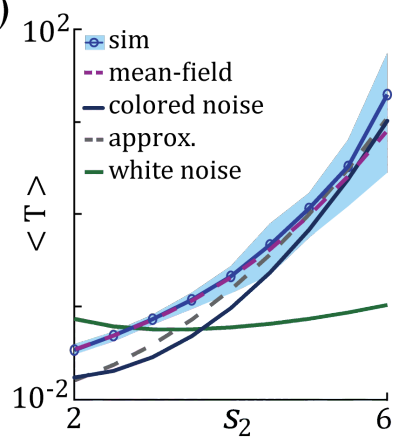

FIG. 4. Separation of timescales and metastable regime. (a) Examples of bistable activity. i,iv - time courses; ii,v - histograms of unit's value across time; iii,vi - histograms of dwell times. (a-i,ii,iii) An example of a probe unit $x_{2}$ with $s_{2}=5$, embedded in a neural network with $N=1000$ units, $N_{1}=N-1$ units with $s_{1}=1$ and $g=1.5$. (a-iv,v,vi) An example of a probe unit driven by white noise. (b) The unified colored noise approximation stationary probability distribution $p\left(x_{2}\right)$ (dark blue curve, Eq. (8), its support excludes the shaded gray area) from the effective potential $U_{\text {eff }}$ (dashed blue curve) captures well the activity histogram (same as (a-ii)); whereas the white noise distribution $p\left(x_{2}\right)$ (dark green curve, obtained from the naive potential $U$, dashed green curve) captures the probe unit's activity (same as (a-v)) when driven by white noise, and deviates significantly from the activity distribution when the probe is embedded in our network. (c) Average dwell times, $\langle T\rangle$, in the bistable states. Simulation results, mean and 95\% CI (blue curve and light blue background, respectively; An example for the full distribution of $T$ is given in (a-iii)). Mean-field prediction (purple curve). The mean first passage time from the unified colored noise approximation (Eq. (10), black curve) captures well the simulated dwell times. An approximation for the unified colored noise (Eq. (11), gray dashed line) achieves good results as well. the white noise average dwell times are significantly different (green curve).

(a)

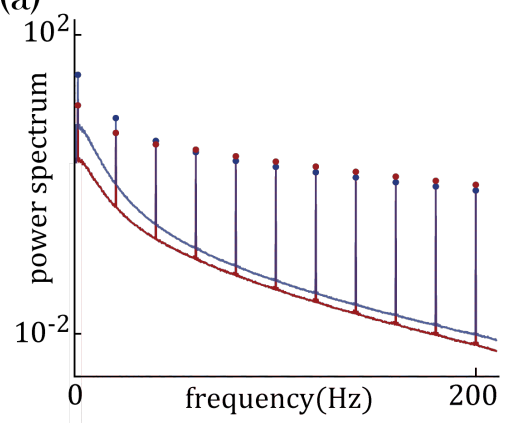

(b)

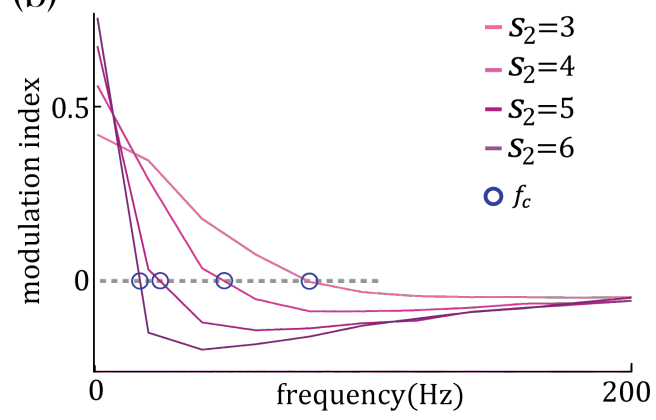

(c) 0.01

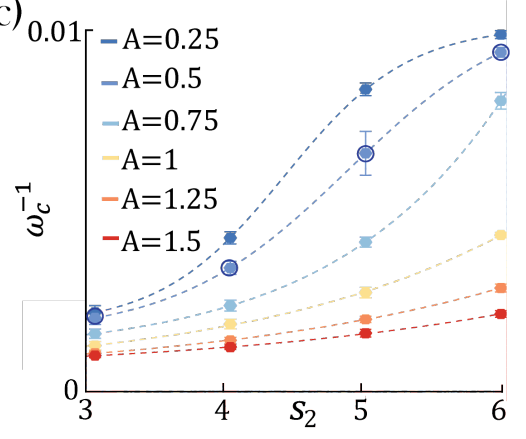

FIG. 5. Network response to broadband input. (a) Power spectrum density of a network driven by time-dependent input comprising a superposition of 11 sinusoidal frequencies (see main text for details). Maroon and navy curves represent average power spectrum density in $s_{1}$ and $s_{2}$ populations, respectively; circles indicate the peak in the power spectrum density amplitudes at each frequency; amplitude $\mathrm{A}=0.5 ; N_{1}=N_{2}=1000, g=3.0, s_{1}=1$ and $s_{2}=4$. (b) Modulation index, Eq. (12), of the power spectrum density amplitudes as a function of frequency in networks with $s_{1}=1$ and various $s_{2}$. The green circles mark the cutoff frequency $f_{c}$ where the modulation index changes sign. (c) Cutoff period, $2 \pi \omega_{c}^{-1}$, as a function of self coupling $s_{2}$ for different input amplitudes. An inversely proportional relation between the cut off period and the amplitude of the broadband signal is present.

two sub-populations, depending on their different intrinsic timescale. We drove each network unit $x_{i}$ with an external broadband stimulus $I_{i}(t)=A \sum_{l=1}^{L} \sin \left(2 \pi f_{l} t+\theta_{i}\right)$ consisting of the superposition of $L$ sinusoidal inputs of different frequencies $f_{l}$ in the range $1-200 \mathrm{~Hz}$, with an equal amplitude $A=0.5$ and random phases $\theta_{i}$. We found that the sub-population with a slow, or fast, intrinsic timescale preferentially entrained its activity with slower, or faster, spectral components of the broadband stimulus respectively (Fig. 5a). We quantified this effect using a spectral modulation index

$$
m(f)=\left[\left(P_{2}(f)-P_{1}(f)\right) /\left(P_{2}(f)+P_{1}(f)\right)\right]
$$

where $P_{\alpha}(f)$ is the power-spectrum peak of subpopulation $\alpha$ at the frequency $f$ (Fig. 5b). A positive, or negative, value of $m(f)$ reveals a stronger, or 
weaker, respectively, entrainment at frequency $f$ in the sub-population $s_{2}$ compared to $s_{1}$. $m(f)$ exhibited a crossover behavior whereby the low frequency component of the input predominantly entrained the slow population $s_{2}$, while the fast component of the input predominantly entrained the fast population $s_{1}$. When fixing $s_{1}=1$ and varying $s_{2}$, we found that the dependence of the crossover frequency $f_{c}$ on $s_{2}$ was strong at low input amplitudes and was progressively tamed at larger input amplitudes (Fig. 5c). This is consistent with the fact that the input amplitude completely suppresses chaos beyond a certain critical value, as previously reported in network's with no self-couplings [15].

Discussion. Previous neural mechanisms for generating multiple timescales of neural activity relied on single cell biophysical properties [21]. Network mechanisms previously proposed were based on self-tuned criticality with anti-hebbian plasticity [22], spatially tuned connectivity gradients [23], or multiple block-structured connectivity [24]. Here, we demonstrated a new robust and biologically plausible network mechanism whereby multiple timescales emerge across units with heterogeneous selfcouplings. In our model, heterogeneous self-couplings can be interpreted as representing the self-interaction of neural clusters of different sizes, consistent with experimental [25-29] and theoretical evidence [30, 31]. In our model, the dynamics of units with large self-couplings, exhibiting slow switching between bistable states, can be captured analytically using the universal colored noise approximation to the Fokker-Planck equation [19, 20], a promising new tool to investigate neural network dynamics. This slow switching regime may underlie the emergence of metastable activity, ubiquitously observed in the population spiking activity of behaving mammals [32-38].

What is the functional relevance of neural circuits exhibiting a reservoir of multiple timescales? The presence of long timescales deeply in the chaotic regime is a new feature of our model which may be beneficial for memory capacity away from the edge of chaos [39]. Moreover, we found that, in our model, time-dependent broadband inputs suppress chaos in a population-specific way, whereby slow (fast) subpopulations preferentially entrain slow (fast) spectral components of the input. This mechanism may thus endow recurrent networks with a natural and robust tool to spatially demix complex temporal inputs [12] as observed in visual cortex [40]. Third, the presence of multiple timescales may be beneficial for performing flexible computations involving simultaneously fast and slow timescales, such as in role-switching tasks [11]; or as observed in time cells in the hippocampus $[41,42]$. A promising direction for future investigation is the exploration of the computational properties of our model in the context of reservoir computing [43] or recurrent networks trained to perform complex cognitive tasks [44].

\section{ACKNOWLEDGMENTS}

We would like to thank G. Mongillo and G. La Camera for discussions. LM was supported by National Institute of Neurological Disorders and Stroke grant R01NS118461 and by National Institute on Drug Abuse grant R01-DA055439 (CRCNS). MS was supported by The Hebrew University of Jerusalem "Emergency response to covid19" grant.
[1] J.-P. Bouchaud, Journal de Physique I 2, 1705 (1992).

[2] E. Almaas, B. Kovacs, T. Vicsek, Z. N. Oltvai, and A.-L. Barabási, Nature 427, 839 (2004).

[3] M. Emmerling, M. Dauner, A. Ponti, J. Fiaux, M. Hochuli, T. Szyperski, K. Wüthrich, J. Bailey, and U. Sauer, Journal of bacteriology 184, 152 (2002).

[4] M. R. Roussel and D. Lloyd, The FEBS Journal 274, 1011 (2007).

[5] M. A. Aon, M. R. Roussel, S. Cortassa, B. O'Rourke, D. B. Murray, M. Beckmann, and D. Lloyd, PLoS One 3, e3624 (2008).

[6] J. D. Murray, A. Bernacchia, D. J. Freedman, R. Romo, J. D. Wallis, X. Cai, C. Padoa-Schioppa, T. Pasternak, H. Seo, D. Lee, et al., Nature neuroscience 17, 1661 (2014).

[7] J. H. Siegle, X. Jia, S. Durand, S. Gale, C. Bennett, N. Graddis, G. Heller, T. K. Ramirez, H. Choi, J. A. Luviano, et al., Biorxiv , 805010 (2019).

[8] R. Gao, R. L. van den Brink, T. Pfeffer, and B. Voytek, Elife 9, e61277 (2020).

[9] A. Bernacchia, H. Seo, D. Lee, and X.-J. Wang, Nature neuroscience 14, 366 (2011).
[10] S. E. Cavanagh, J. D. Wallis, S. W. Kennerley, and L. T. Hunt, Elife 5, e18937 (2016).

[11] K. Iigaya, Y. Ahmadian, L. P. Sugrue, G. S. Corrado, Y. Loewenstein, W. T. Newsome, and S. Fusi, Nature communications 10, 1 (2019).

[12] N. Perez-Nieves, V. C. Leung, P. L. Dragotti, and D. F. Goodman, bioRxiv , 2020 (2021).

[13] H. Sompolinsky, A. Crisanti, and H.-J. Sommers, Physical review letters 61, 259 (1988).

[14] M. Stern, H. Sompolinsky, and L. Abbott, Physical Review E 90, 062710 (2014).

[15] K. Rajan, L. Abbott, and H. Sompolinsky, Physical Review E 82, 011903 (2010).

[16] M. A. Buice and C. C. Chow, Journal of Statistical Mechanics: Theory and Experiment 2013, P03003 (2013).

[17] M. Helias and D. Dahmen, Statistical Field Theory for Neural Networks (Springer).

[18] Y. Ahmadian, F. Fumarola, and K. D. Miller, Physical Review E 91, 012820 (2015).

[19] P. Hänggi and P. Jung, Advances in chemical physics 89, 239 (1995).

[20] P. Jung and P. Hänggi, Physical review A 35, 4464 (1987). 
[21] J. Gjorgjieva, G. Drion, and E. Marder, Current opinion in neurobiology 37, 44 (2016).

[22] M. O. Magnasco, O. Piro, and G. A. Cecchi, Physical review letters 102, 258102 (2009).

[23] R. Chaudhuri, A. Bernacchia, and X.-J. Wang, elife 3, e01239 (2014).

[24] J. Aljadeff, M. Stern, and T. Sharpee, Physical review letters 114, 088101 (2015).

[25] S. Song, P. J. Sjöström, M. Reigl, S. Nelson, and D. B. Chklovskii, PLoS Biol. 3, e68 (2005).

[26] R. Perin, T. K. Berger, and H. Markram, Proc. Natl. Acad. Sci. U. S. A. 108, 5419 (2011).

[27] W.-C. A. Lee, V. Bonin, M. Reed, B. J. Graham, G. Hood, K. Glattfelder, and R. Clay Reid, "Anatomy and function of an excitatory network in the visual cortex," (2016).

[28] R. Kiani, C. J. Cueva, J. B. Reppas, D. Peixoto, S. I. Ryu, and W. T. Newsome, Neuron 85, 1359 (2015).

[29] J.-e. K. Miller, I. Ayzenshtat, L. Carrillo-Reid, and R. Yuste, Proceedings of the National Academy of Sciences 111, E4053 (2014).

[30] A. Litwin-Kumar and B. Doiron, Nat Neurosci 15, 1498 (2012).

[31] D. Wyrick and L. Mazzucato, Journal of Neuroscience 41, 3988 (2021).

[32] M. Abeles, H. Bergman, I. Gat, I. Meilijson, E. Seidemann, N. Tishby, and E. Vaadia, Proc Natl Acad Sci USA 92, 8616 (1995).

[33] L. M. Jones, A. Fontanini, B. F. Sadacca, P. Miller, and D. B. Katz, Proc Natl Acad Sci U S A 104, 18772 (2007).

[34] L. Mazzucato, A. Fontanini, and G. La Camera, The Journal of Neuroscience 35, 8214 (2015).

[35] L. Mazzucato, G. La Camera, and A. Fontanini, Nature neuroscience 22, 787 (2019).

[36] S. Recanatesi, U. Pereira, M. Murakami, Z. Mainen, and L. Mazzucato, bioRxiv (2020).

[37] T. A. Engel, N. A. Steinmetz, M. A. Gieselmann, A. Thiele, T. Moore, and K. Boahen, Science 354, 1140 (2016).

[38] N. Kadmon Harpaz, D. Ungarish, N. G. Hatsopoulos, and T. Flash, Cerebral Cortex 29, 1619 (2019).

[39] T. Toyoizumi and L. Abbott, Physical Review E 84, 051908 (2011).

[40] A. Mazzoni, S. Panzeri, N. K. Logothetis, and N. Brunel, PLoS Comput Biol 4, e1000239 (2008).

[41] B. J. Kraus, R. J. Robinson II, J. A. White, H. Eichenbaum, and M. E. Hasselmo, Neuron 78, 1090 (2013).

[42] M. W. Howard, C. J. MacDonald, Z. Tiganj, K. H. Shankar, Q. Du, M. E. Hasselmo, and H. Eichenbaum, Journal of Neuroscience 34, 4692 (2014).

[43] D. Sussillo and L. F. Abbott, Neuron 63, 544 (2009).

[44] G. R. Yang, M. R. Joglekar, H. F. Song, W. T. Newsome, and X.-J. Wang, Nature neuroscience 22, 297 (2019).

[45] H. Sompolinsky and A. Zippelius, Phys. Rev. B 25, 6860 (1982).

[46] A. Crisanti and H. Sompolinsky, Phys. Rev. A 36, 4922 (1987).

[47] P. C. Martin, E. D. Siggia, and H. A. Rose, Phys. Rev. A 8, 423 (1973).

\section{Appendix - Dynamical regions of networks with identical self-couplings, a summary}

It is constructive to quickly survey the results of Stern et al. [14] who studied the special case of including a single value self-coupling $s$ for all clusters in the network, $P\left(s_{i}\right)=\delta_{s, s_{i}}$. In this case the dynamics of all units in the network follow:

$$
\frac{d x_{i}}{d t}=-x_{i}+s \tanh \left(x_{i}\right)+g \sum_{i=1}^{N} J \phi\left(x_{j}\right),
$$

Two variables determine the network dynamics, the network gain $g$ and the self-coupling value $s$. The network gain $g$ defines the strength of the network impact on its units. It brings the network into chaotic activity, without self-coupling $(s=0)$, for values $g>1$ [13]. The self-coupling $s$ generates bi-stability. Without network impact $(g=0)$ the dynamical equation 13 for each unit becomes

$$
\frac{d x_{i}}{d t}=-x_{i}+s \tanh \left(x_{i}\right)
$$

which has two stable solutions for $s>1$ (Appendix Fig. 1a), both at $x \neq 0$. For $s<1$ (Appendix Fig. 1b) a single stable solution exists at $x=0$.

When small values of network gain $g$ are introduced to the network dynamics, Eq. (13), with identical bi-stable units $(s>1)$, each unit solution jitters around one of its two possible fixed points. After an irregular activity the network settle into a stable fixed point. This generates a region of transient irregular activity with stable fixed points (Appendix Fig. 1c). As $g$ increases and $s$ decreases, different possible fixed point configurations lose their stability (as a result, the typical time spent in the transient activity increases). When the critical line $s_{c} \approx 1+0.157 \ln (0.443 g+1)$ is crossed, no fixed point remains stable and the network activity becomes chaotic [14]. The "last" stable fixed point at the transition line has a unique configuration with all unit values located farthest from $x=0$ (Appendix Fig. 1a, light green lines). Additional decrease of $s$ and $g$ leads to a region where any initial activity of the network decays and the trivial solution $\left(x_{i}=0\right.$ for all $\left.i\right)$ is stable (Appendix Fig. 1c).

\section{Appendix - Mean field theory with multiple self-couplings}

We derive the dynamic mean-field theory in the limit $N \rightarrow \infty$ by using the moment generating functional [45, 46]. For the derivation we follow the Martin-Siggia-RoseDe Dominicis-Janssen path integral approach formalism [47] as appears extensively in [17], we borrow their notations as well. For our model, Eq. (1), the moment generating functional is given by:

$$
\begin{aligned}
Z=\int \mathcal{D} \tilde{x} \mathcal{D} x \exp & {\left[\int d t \sum_{i=1}^{N} \tilde{x}_{i}(t)\left(\left(\partial_{t}+1\right) x_{i}(t)-s_{i} \phi\left(x_{i}(t)\right)\right)\right.} \\
& \left.-\sum_{j \neq i} \tilde{x}_{i}(t) J_{i j} \phi\left(x_{j}(t)\right)\right]
\end{aligned}
$$



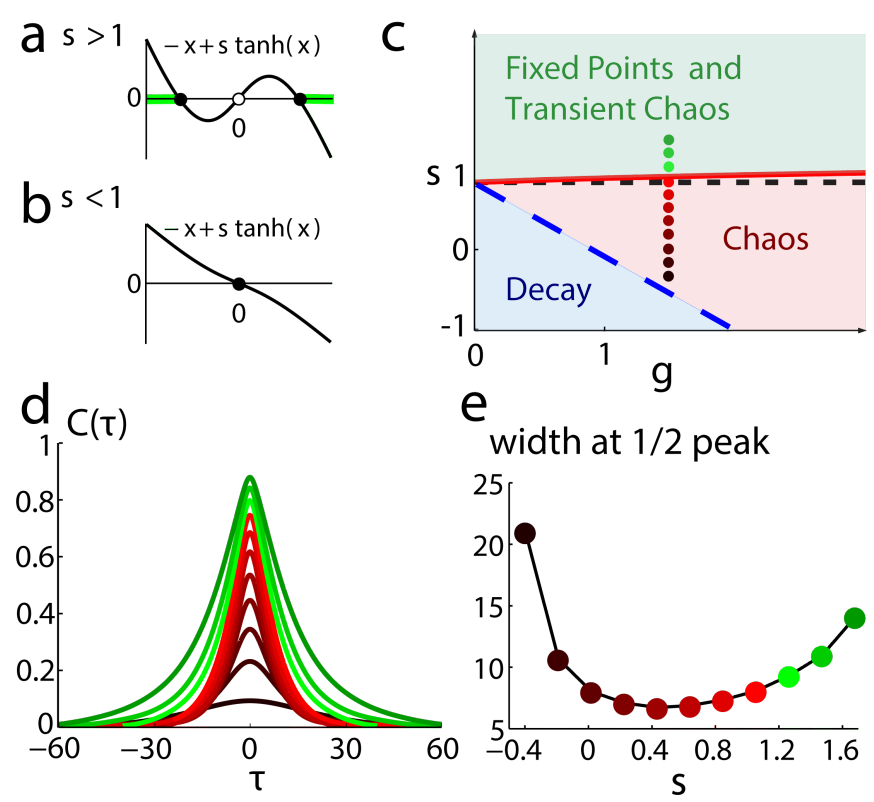

FIG. 6. Network dynamics with identical self-couplings, adopted from [14]. a,b) Graphical solutions to Eq. (14). a) For $s>1$ there are two stable non zero solutions (full black circles) and an unstable solution at zero (open black circle). The green background over the $x$-axis denotes the regions of allowed activity values at the stable fixed point on the transition line to chaos (solid red curve in (c)). b) For $s<1$ there is a single stable solution (full black circle) at zero. c) Regions of the network dynamics over a range of $s$ and $g$ values. Below the long dashed blue line any initial activity in the network decays to zero. Above the solid red curve, the network exhibits transient irregular activity that eventually settles into one out of a number of possible nonzero stable fixed points. In the region between these two curves, the network activity is chaotic. Colored circles denote, according to their locations on the phase diagram and with respect to their colors, the values of $s$ (ranging from 1.6 to 0.4 in steps of 0.2 ) and $g=1.5$, used for the autocorrelation functions $C(\tau)$ in (d) ${ }^{\text {a }}$. e) Widths at $1 / 2$ peak (values of $\tau$ 's in the main text notation) of the autocorrelation functions in (d).

a Corrected version

where $\mathcal{D} x=\prod_{i} \mathcal{D} x_{i}$ and $\mathcal{D} \tilde{x}=\prod_{i} \mathcal{D} \tilde{x}_{i} / 2 \pi i$. To start, we calculate $\langle Z(J)\rangle_{J}$. We take advantage of the selfaveraging nature of our model, particularly by averaging over the quenched disorder, $J$. The couplings, $J_{i j}$, are i.i.d. variables extracted from a normal distribution and appear only in the last term in (15). We hence focus our current calculation step on that term, and we derive the result to the leading term in $N$, yielding:

$$
\begin{aligned}
& \int \prod_{i \neq j} d J_{i j} \sqrt{\frac{N}{2 \pi g^{2}}} \exp \left[-\frac{J_{i j}^{2} N}{2 g^{2}}\right] \exp \left[-\int d t \tilde{x}_{i}(t) J_{i j} \phi\left(x_{j}(t)\right)\right] \\
& =\exp \left[\frac{1}{2} \int d t d t^{\prime}\left(\sum_{i} \tilde{x}_{i}(t) \tilde{x}_{i}\left(t^{\prime}\right)\right)\left(\frac{g^{2}}{N} \sum_{j} \phi\left(x_{j}(t)\right) \phi\left(x_{j}\left(t^{\prime}\right)\right)\right)\right] .
\end{aligned}
$$

The result above suggests that all the units in our net- work are coupled to one another equivalently (by being coupled only to sums that depend on all units' activity). To further decouple the network, we define the quantity

$$
Q_{1}\left(t, t^{\prime}\right) \equiv \frac{g^{2}}{N} \sum_{j} \phi\left(x_{j}(t)\right) \phi\left(x_{j}\left(t^{\prime}\right)\right)
$$

We enforce this definition by multiplying the disordered averaged moment generating functional with the appropriate Dirac delta function, $\delta$, in its integral form:

$$
\begin{aligned}
1 & =\int d Q_{1} \delta\left[-\frac{N}{g^{2}} Q_{1}+\sum_{j} \phi\left(x_{j}(t)\right) \phi\left(x_{j}\left(t^{\prime}\right)\right)\right] \\
& =\int d Q_{1} d Q_{2} \exp Q_{2}\left[-\frac{N}{g^{2}} Q_{1}+\sum_{j} \phi\left(x_{j}(t)\right) \phi\left(x_{j}\left(t^{\prime}\right)\right)\right]
\end{aligned}
$$

where $d Q_{2}$ is an integral over the imaginary axis (including its $1 /(2 \pi i)$ factor $)$. We can now rewrite the disordered averaged moment generating functional, using (16) to replace its last term, the definition of $Q_{1}$, and with multiplying the functional by the $\delta$ function above. All together we get:

$$
\begin{aligned}
\langle Z(J)\rangle_{J} & =\int d Q_{1} d Q_{2} \exp \left[-\frac{N}{g^{2}} \int d t d t^{\prime} Q_{1} Q_{2}+N \sum_{\alpha \in A} n_{\alpha} \ln \left[Z_{\alpha}\right]\right] \\
Z_{\alpha} & =\int \mathcal{D} \tilde{x}_{\alpha} \mathcal{D} x_{\alpha} \exp \left[\int d t \tilde{x}_{\alpha}(t)\left(\left(\partial_{t}+1\right) x_{\alpha}(t)-s_{\alpha} \phi\left(x_{\alpha}(t)\right)\right)+\right. \\
& +\frac{1}{2} \int d t d t^{\prime} \tilde{x}_{\alpha}(t) Q_{1}\left(t, t^{\prime}\right) \tilde{x}_{\alpha}\left(t^{\prime}\right)+ \\
& \left.+\int d t d t^{\prime} \phi\left(x_{\alpha}(t)\right) Q_{2}\left(t, t^{\prime}\right) \phi\left(x_{\alpha}\left(t^{\prime}\right)\right)\right]
\end{aligned}
$$

with $n_{\alpha}=N_{\alpha} / N$ the fraction of units with self-couplings $s_{\alpha}$ across the population, for $\alpha \in A$. In the expression above we made use of the fact that $Q_{1}$ and $Q_{2}$, now in a role of auxiliary fields, couple to sums of the fields $x_{i}^{2}$ and $\phi_{i}^{2}$ and hence the generating functional for $x_{i}$ and $\tilde{x}_{i}$ can be factorized with identical multiplications of $Z_{\alpha}$. Note that in our network, due to the dependency on $s_{i}$, $x_{i}$-S are equivalent as long as $s_{i}$-S are equivalent. Hence, the factorization is for $Z_{\alpha}$ for all $x_{i}$ with $s_{i}=s_{\alpha}$. Now each factor $Z_{\alpha}$ includes the functional integrals $\mathcal{D} x_{\alpha}$ and $\mathcal{D} \tilde{x}_{\alpha}$ for a single unit with self-coupling $s_{\alpha}$.

In the large $N$ limit we evaluate the auxiliary fields in (17) by the saddle point approach (we note variable valued at the saddle point by $*$ ), obtaining:

$$
0=\frac{\delta}{\delta Q_{1,2}}\left[-\frac{1}{g^{2}} \int d t d t^{\prime} Q_{1} Q_{2}+\sum_{\alpha \in A} n_{\alpha} \ln \left[Z_{\alpha}\right]\right]
$$


and yielding the saddle point values $\left(Q_{1}^{*}, Q_{2}^{*}\right)$ :

$$
\begin{aligned}
0 & =-\frac{1}{g^{2}} Q_{1}^{*}\left(t, t^{\prime}\right)+\left.\sum_{\alpha \in A} \frac{n_{\alpha}}{Z_{\alpha}} \frac{\partial Z_{\alpha}}{\partial Q_{2}\left(t, t^{\prime}\right)}\right|_{Q^{*}} \\
& \Leftrightarrow Q_{1}^{*}\left(t, t^{\prime}\right)=g^{2} \sum_{\alpha \in A} n_{\alpha}\left\langle\phi\left(x_{\alpha}(t)\right) \phi\left(x_{\alpha}\left(t^{\prime}\right)\right)\right\rangle \equiv g^{2} C(\tau),
\end{aligned}
$$

$$
\begin{aligned}
0 & =-\frac{1}{g^{2}} Q_{2}^{*}\left(t, t^{\prime}\right)+\left.\sum_{\alpha \in A} \frac{n_{\alpha}}{Z_{\alpha}} \frac{\partial Z_{\alpha}}{\partial Q_{1}\left(t, t^{\prime}\right)}\right|_{Q^{*}} \\
& \Leftrightarrow Q_{2}^{*}\left(t, t^{\prime}\right)=\frac{g^{2}}{2} \sum_{\alpha \in A} n_{\alpha}\left\langle\tilde{x}_{\alpha}(t) \tilde{x}_{\alpha}\left(t^{\prime}\right)\right\rangle=0,
\end{aligned}
$$

where $C(\tau)$, with $\tau=f\left(t, t^{\prime}\right)$, represents the average autocorrelation function of the network (as was defined in the main text). The second saddle point $Q_{2}^{*}=0$ vanishes due to $\left\langle\tilde{x}_{\alpha}(t) \tilde{x}_{\alpha}\left(t^{\prime}\right)\right\rangle=0$ as can be immediately extended from $[17,45]$. The action at the saddle point reduces to the sum of actions for individual, non-interacting units with self-coupling $s_{\alpha}$. All units are coupled to a common external field $Q_{1}^{*}$. Inserting the saddle point values back into Eq. (17), we obtain $Z^{*}=\prod_{\alpha}\left(Z_{\alpha}^{*}\right)^{N_{\alpha}}$ where

$$
\begin{aligned}
Z_{\alpha}^{*} \sim & \int \mathcal{D} \tilde{x}_{\alpha} \mathcal{D} x_{\alpha} \exp \sum_{\alpha \in A}\left(\int d t \tilde { x } _ { \alpha } ( t ) \left(\left(\partial_{t}+1\right) x_{\alpha}(t)\right.\right. \\
& \left.\left.-s_{\alpha} \phi\left(x_{\alpha}(t)\right)\right)+\frac{g^{2}}{2} \int d t d t^{\prime} \tilde{x}_{\alpha}(t) C(\tau) \tilde{x}_{\alpha}\left(t^{\prime}\right)\right) .
\end{aligned}
$$

Thus in the large $N$ limit the network dynamics are reduced to those of a number of $A$ units $x_{\alpha}(t)$, each represents the sub-population with self-couplings $s_{\alpha}$ and follows dynamics governed by

$$
\frac{d}{d t} x_{\alpha}(t)=-x_{\alpha}(t)+s_{\alpha} \phi\left[x_{\alpha}(t)\right]+\eta(t)
$$

for all $\alpha \in A$ and where $\eta(t)$ is a Gaussian mean field with autocorrelation

$$
\left\langle\eta(t) \eta\left(t^{\prime}\right)\right\rangle=g^{2} \sum_{\alpha \in A} n_{\alpha}\left\langle\phi\left(x_{\alpha}(t)\right) \phi\left(x_{\alpha}\left(t^{\prime}\right)\right)\right\rangle .
$$

The results above can be immediately extended for the continuous case of self-coupling distribution $P(s)$ yielding:

$$
\left\langle\eta(t) \eta\left(t^{\prime}\right)\right\rangle=g^{2} \int p(s) \phi(x(s, t)) \phi\left(x\left(s, t^{\prime}\right)\right) d s
$$

with $p(s)$ the density function of the self-couplings distribution in the network and the units dynamics dependent on their respective self-couplings:

$$
\frac{d}{d t} x(s, t)=-x(s, t)+s \phi[x(s, t)]+\eta(t) .
$$

